\title{
Liver Disease in Alpha-1 Antitrypsin Deficiency: Current Approaches and Future Directions
}

\author{
Ellen L. Mitchell ${ }^{1,2}$ - Zahida Khan ${ }^{1,2,3,4,5}$
}

Published online: 10 July 2017

(C) Springer Science+Business Media, LLC 2017

\begin{abstract}
Purpose of Review The aim of the study is to review the liver disease caused by alpha-1 antitrypsin deficiency (A1ATD), including pathogenesis, epidemiology, diagnostic testing, and recent therapeutic developments.

Recent Findings Therapeutic approaches target several intracellular pathways to reduce the cytotoxic effects of the misfolded mutant globular protein (ATZ) on the hepatocyte. These include promoting ATZ transport out of the endoplasmic reticulum (ER), enhancing ATZ degradation, and preventing ATZ globule-aggregation.

Summary A1ATD is the leading genetic cause of liver disease among children. It is a protein-folding disorder in which toxic insoluble ATZ proteins aggregate in the ER of hepatocytes leading to inflammation, fibrosis, cirrhosis, and increased risk of hepatocellular carcinoma. The absence of the normal A1AT
\end{abstract}

This article is part of the Topical Collection on Pathobiology of Orphan Diseases

Zahida Khan

zahida.khan@chp.edu

Ellen L. Mitchell

ellen.mitchell2@chp.edu

1 Division of Pediatric Gastroenterology, Hepatology, and Nutrition, Children's Hospital of Pittsburgh of UPMC, 4401 Penn Avenue, Faculty Pavilion 6th Fl, Pittsburgh, PA 15224-1334, USA

2 Department of Pediatrics, University of Pittsburgh School of Medicine, Pittsburgh, PA, USA

3 Department of Pathology, University of Pittsburgh School of Medicine, Pittsburgh, PA, USA

4 McGowan Institute for Regenerative Medicine, University of Pittsburgh School of Medicine, Pittsburgh, PA, USA

5 Pittsburgh Liver Research Center, University of Pittsburgh School of Medicine, Pittsburgh, PA, USA serum protein also predisposes patients to pan lobar emphysema as adults. At this time, the only approved therapy for A1ATD-associated liver disease is orthotopic liver transplantation, which is curative. However, there has been significant recent progress in the development of small molecule therapies with potential both to preserve the native liver and prevent hepatotoxicity.

Keywords PiZ mouse $\cdot$ Autophagy $\cdot$ ATZ $\cdot$ Liver transplantation $\cdot$ SERPINA1 $\cdot$ Bile acid

\section{Introduction}

Alpha-1 antitrypsin deficiency (A1ATD) is a common inherited cause of liver disease, with the most severe mutation found in 1:3500 live births and currently 180,000 individuals worldwide [1]. In children, A1ATD is the most frequent genetic etiology for pediatric liver disease and transplantation [2]. Caused by a mutation in chromosome 14, the disease is inherited in an autosomal recessive manner with codominant expression [3]. There are over 100 variants of the alpha-1 antitrypsin (A1AT) gene (SERPINA1) [4], which can be influenced by other genetic and environmental factors [5], leading to marked variation in the onset and severity of disease [6]. The most common variant forms were named as Fast (F), Medium (M), Slow (S), and Very Slow (Z) based on molecular migration speed on an isoelectric $\mathrm{pH}$ gradient, with differences in charges attributed to the amino acid composition [7]. The normal gene product is PiM, and the most common mutations are PiS and PiZ, which express $50-60$ and $10-20 \%$ of the A1AT protein, respectively. More rare deficient alleles account for $<5 \%$ of patients with the disease [8].

A1ATD is a proteinopathy (a disease resulting from the abnormal synthesis, folding, post-translational modification, 
or deposition of protein in cells or tissues) that results in proteotoxicity (impaired cellular function by aggregation of misfolded protein). Normal human A1AT is a $52-\mathrm{kDa}$ glycoprotein of the serpin (serine proteinase inhibitor) family, predominantly produced in the liver and released into the blood. In affected patients, circulating levels of mutant A1AT are $\leq 15 \%$ of normal protein levels. A1ATD is unique in that it is a proteinopathy that impacts the lungs and the liver by different mechanisms [9]. In the lungs, it is a loss-of-function mutation, where A1AT is an inhibitor of neutrophil elastase. Emphysema results when A1AT is not present to inhibit the serine proteinase, allowing it to freely destroy the lung tissue [10]. The liver disease associated with A1ATD is a gain-of-toxic function mechanism. The misfolded insoluble globular proteins (ATZ) accumulate in the endoplasmic reticulum (ER), leading to hepatic fibrosis and even hepatocellular carcinoma (HCC) [11].

While liver transplantation is the only curative treatment available at this time, there have been several developments towards alternative therapies that would preserve the native liver. Autophagy has been a major target for therapeutic strategies because of the key role that it plays in the intracellular degradation of ATZ [12]. Both physical modifications and drug targets are being utilized to explore mechanisms to increase autophagy. Genetic therapies are also being developed, either to enhance autophagy or directly modify the mutated gene.

\section{Diagnosis}

The diagnosis of A1ATD is confirmed with laboratory analysis. Serum levels of A1AT can result in a false positive result because it is also an acute phase reactant and therefore elevated in the setting of inflammation. Phenotyping by isoelectric focusing is the gold standard of diagnosis [13]. Although liver biopsy is not required for diagnosis, it may be helpful in difficult cases and/or for prognostication. Histologically, insoluble ATZ proteins are characterized by globules which stain bright pink with periodic acid-Schiff's reagent, but unlike glycogen granules, they are resistant to diastase treatment (Fig. 1a). Anti-human A1AT antibody can also confirm the presence of ATZ globules (Fig. 1b). Electron microscopy studies can identify multiple globular inclusions within dilated, congested rough ER cisterns, adjacent to secondary lysosomes (Fig. 1c-d) [14]. As the ATZ globules accumulate, chronic hepatocyte injury from ER stress and proteotoxicity leads to mitochondrial damage and impaired protein clearance [11].

Data combined from multiple studies have estimated the incidence of A1ATD as 1:1600 to 1:3500 cases, an incidence comparable to that of cystic fibrosis [15]. A1ATD is likely an underdiagnosed condition because of its nonspecific presentation. It has been estimated that only $3.8 \%$ of severely affected individuals are currently identified [16], and there is an average delay in diagnosis of 5.6 years ( \pm 8.5 years) $[17,18]$. There have been attempts to improve the identification of patients both with
Fig. 1 Liver histopathology of A1AT deficiency. a PASD stain highlights ATZ globules (pink) in a human explanted liver from a pediatric patient with severe liver disease $(\times 400$, scale bar $50 \mu \mathrm{M})$. b Anti-human A1AT immunohistochemistry of ATZ globules from same patient as a $(\times 400$, scale bar $50 \mu \mathrm{M})$. c, d EM of pediatric liver explant shows aggregation of insoluble proteinaceous material $(*)$ in rough ER $(\mathbf{c}, \times 40,000)$ and accumulation of autophagolysosomes (d, $\times 25,000)$. $M$ mitochondria, $N$ nucleus, $F$ fat. Images reproduced with permission from Dr. Sarangarajan Ranganathan (a, b) and Dr. Donna B. Stolz (c, d)
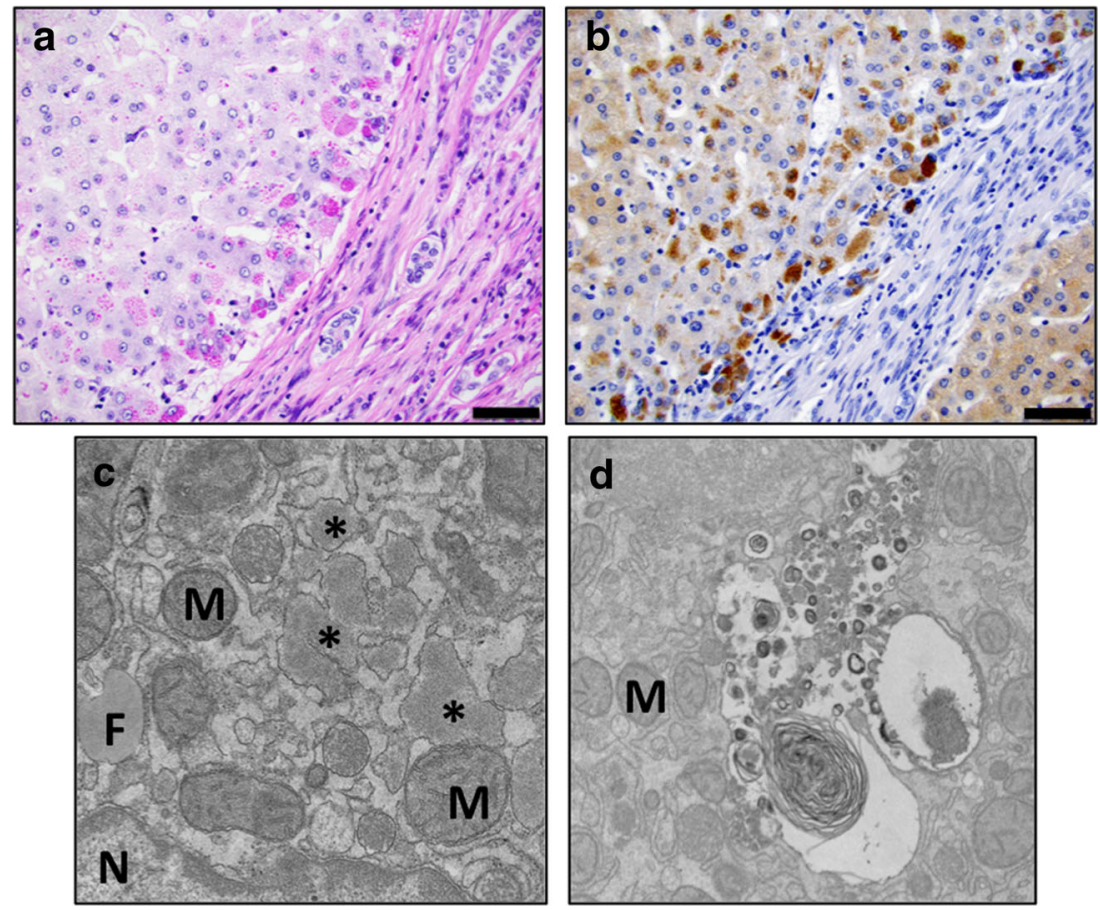
liver and lung disease by voluntary screening [19]. Universal newborn screening has been implemented in Sweden. Out of the 200,000 Swedish newborns reported to be tested, 127 were found to be PIZZ homozygotes. As this cohort was followed and compared to healthy controls, they were found to not only have more clinically apparent liver disease but also more laboratory abnormalities and symptoms that otherwise may have gone undetected [20, 21]. Even so, only 8-10\% of these PiZZ homozygotes developed severe liver disease in the first four decades of life, suggesting the co-existence of other unknown disease modifiers [22]. As therapies evolve and more evidence emerges to support the benefits of screening, other countries, including the USA, may consider adding A1ATD to the newborn screen [15, 23, 24].

While there is a clear indication to screen patients with liver disease of unknown etiology, there may also be benefit to screening select patients that carry an established diagnosis of any liver disease. Although A1ATD is typically diagnosed in patients that are homozygous for the mutation, Perlmutter and colleagues found that a significant portion of patients transplanted for A1ATD were actually heterozygous for the mutation (mostly PiMZ), but found to have another "second hit" insult that could have caused or contributed to more rapid progression of endstage liver disease $[25,26]$. These risk factors include infections (CMV and hepatitis B and C), genetic disorders (cystic fibrosis), and other co-morbidities (obesity, alcoholism) [25, 27, 28]. Environmental factors such as cigarette smoking can propagate ongoing lung injury [29-32]. Screening for patients that carry one or both mutated alleles has been shown to have a positive impact, by identifying those patients requiring genetic counseling, anticipatory guidance, and lifestyle modifications, along with closer monitoring for progressive liver and lung dysfunction [15]. In addition to these clinical variables, a small subset of genetic modifiers for disease susceptibility have also been reported. These include single nucleotide polymorphisms (SNPs) in the SERPINA1 gene, along with critical ER proteins (calnexin, ERmanI) [33-37]. Interestingly, over two-thirds of all patients requiring liver transplantation for A1ATD are male, suggesting sex-dependent phenotypic variations [25]. The latter has also been demonstrated in transgenic mouse models of A1ATD, where female PiZ mice have significantly less ATZ globules compared to males at baseline, but will develop the classical form of A1ATD-associated liver disease if they are treated with testosterone [38]. Overall, these genetic and environmental disease modifiers may be useful to aid in the treatment and prognosis of A1ATD liver disease.

\section{Current Management}

Although intravenous augmentation therapy of pooled human A1AT protein is available as a supportive therapy for adults with lung disease, it is not considered appropriate treatment for liver- affected patients. In patients with severe liver disease who are awaiting transplantation, morbidity increases with the complications of cholestasis, portal hypertension, and cirrhosis, all of which lead to end-stage liver failure. These patients can succumb to life-threatening variceal bleeding, coagulopathy, and encephalopathy. Proteotoxic stress also triggers malignant transformation to HCC. Liver transplantation is the only curative therapy for A1ATD liver disease at this time [39]. A1ATD accounts for $3.51 \%$ of pediatric liver transplants, with 1-, 3-, and 5-year graft survivals of 84,81 , and $78 \%$ respectively, and patient survivals of 92,90 , and $90 \%$ respectively [40]. In contrast to children, a recent analysis of three US transplantation databases reported that $77.2 \%$ of 1677 total liver transplants with a reported diagnosis of A1ATD were adults (peak age 50-64), similar to other agedependent degenerative disorders [25]. Recipients express the donor phenotype; therefore, the progression of lung disease is halted, and they do not experience recurrence of liver disease [40]. Overall, both adults and children with A1ATD appear to have excellent survival post-liver transplant [40]. Nevertheless, promising new small molecule therapies (Fig. 2) can be developed to ease the public health burden from end-stage liver failure and limited availability of donor organs.

\section{Evolving Therapies}

\section{Chaperones}

One approach to reduce the accumulation ATZ globules is to increase their transport out of the ER with chemical chaperones. Phenylbutyrate (PBA) is already used as an ammonia scavenger in urea cycle disorders and has been used as a chaperone in several other diseases [41-47]. Utilizing the CJZ12B cell line genetically engineered to over-express ATZ proteins, PBA resulted in a fivefold increase in the secretion of A1AT into the extracellular fluid. The secreted A1AT was likely functionally active as a serpin, because it was able to form a complex with elastase. PiZ transgenic mice, which over-express human ATZ to recapitulate the liver disease found in A1ATD, were used as an in vivo model with similar findings [48]. Unfortunately, when PBA was given in an open-label study to 10 patients with A1ATD for 2 weeks, there was no increase in serum protein levels of A1AT, and metabolic side effects were noted [49]. Clearly, more research is needed to clarify the therapeutic role of such chaperones in protein-folding, trafficking, and secretion of soluble A1AT.

\section{Autophagy-Enhancing Agents}

Autophagy allows for directed removal of dysfunctional intracellular proteins, and therefore is an appropriate target for therapy to process insoluble ATZ globules. Several medications currently used for other diseases have been found to also 


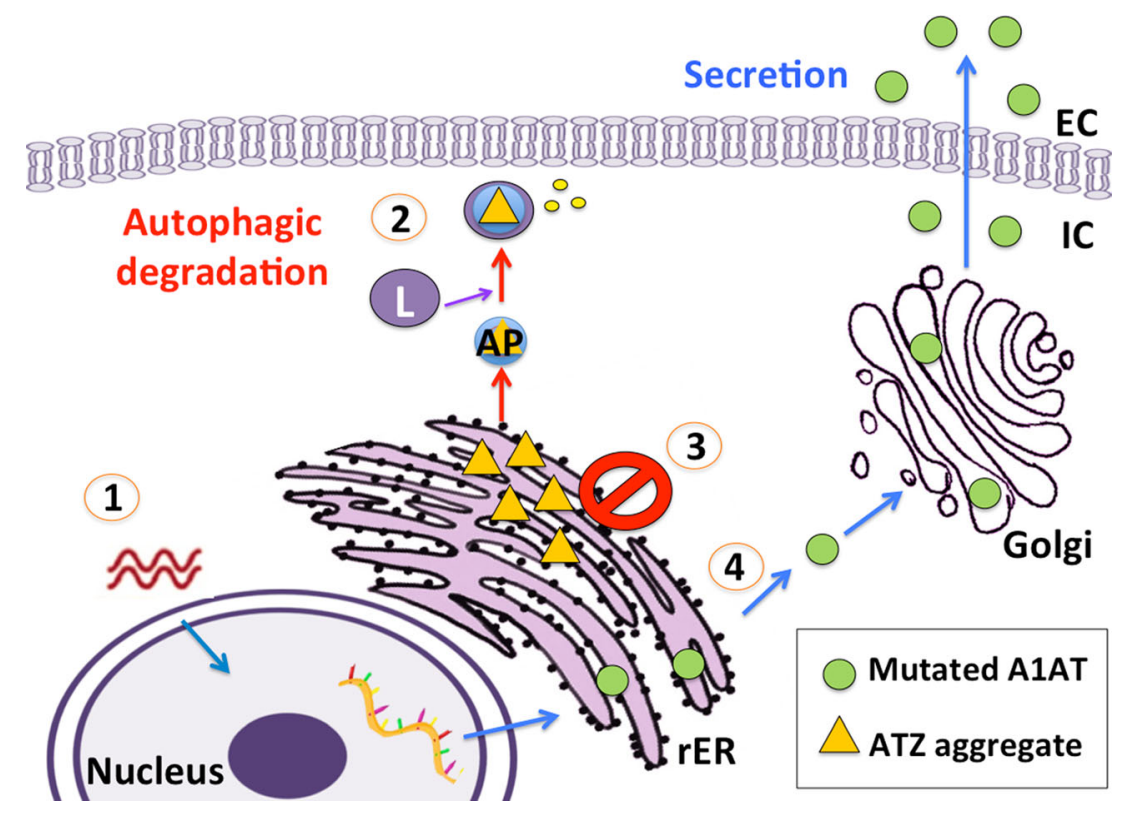

Fig. 2 Pathogenesis of liver disease in A1AT deficiency and potential pharmacologic interventions. Mutated A1AT is produced in the nucleus, transported to the ER where it aggregates, and forms ATZ globules. Therapeutic targets focus on several common pathways. (1) genetic modification: RNAi, viral gene transfer, and stem cell therapy. (2) Increase autophagy: carbamazepine, rapamycin, and bile acids. (3) block polymerization: nucleoside analog S-(4-nitrobenzyl)-6thioguanosine, single chain antibody fragments $(\mathrm{scFv})$, and compounds that bind to the allosteric cavity of A1AT. (4) increase secretion: phenylbutyrate. $I C$ intercellular, $E C$ extracellular, $r E R$ rough endoplasmic reticulum, $A P$ autophagosome, $L$ lysosome (modified from Khan 2016 [2]) enhance autophagy. Caenorhabditis elegans have been used as a high-throughput and high-content live animal screening model because they are small, easy to grow, and transparent, allowing proteins to be fluorescently labeled in order to track outcomes [50-52]. Although C. elegans is a simple roundworm that lacks respiratory, circulatory, and hepatobiliary systems, it can be genetically engineered to express wild-type (ATM) or mutant forms (ATZ) of the human SERPINA1 gene fused to green fluorescent protein (GFP) [53]. These animals secrete soluble ATM-GFP but retain polymerized ATZ-GFP protein within dilated ER cisternae. Utilizing a library of 1280 pharmacologically active compounds, C. elegans demonstrated a decrease in ATZ-GFP with the application of cantharidin, tamoxifen, fluphenazine, and pimozide [54]. Although these results are preliminary, they encourage the screening of approved medications with known safety profiles to expedite developments in treatment.

Rapamycin and carbamazepine are other examples of medications used for alternate diseases that could be repurposed to treat A1ATD by targeting autophagic pathways. Rapamycin is an mTOR inhibitor used for immunosuppression, but it has also been shown to enhance autophagy $[55,56]$. Daily dosing had no impact in PiZ mice; however, when the same dosage was consolidated to weekly administration, there was a significant increase in autophagic vacuoles with a concomitant decrease in ATZ protein, and improved hepatic fibrosis. There was also a decrease in compensatory hepatocellular proliferation, which is another known response to hepatocyte damage $[38,57]$. As a drug that is already approved by the FDA, rapamycin may be an appropriate target for further trials; however, concerns exist about this drug's potential lung toxicity, which though rare, may aggravate underlying lung dysfunction in patients with A1ATD.

In contrast, carbamazepine (CBZ) is a well-known antiepileptic and mood stabilizer with an extensive safety profile. CBZ has been shown to enhance the degradation of mutant ATZ both through autophagic and proteosomal mechanisms [58]. The reduction in ATZ was seen both in the HeLa inducible cell line HTO/Z and in PiZ x GFP-LC3 mice, which allows for intracellular tracking of the degraded human ATZ proteins in autophagosomes [58]. Interestingly, serum concentrations of human A1AT in PiZ mice were not significantly affected by CBZ treatment, arguing against any effect on secretion of ATZ in vivo [58]. Nevertheless, as a pre-clinical model, the PiZ transgenic mice did show a reduction in hepatic fibrosis with CBZ therapy. Of note, the projected dose needed in humans for this hepatoprotective effect is more than 10 times the current approved dose of CBZ [58]. However, mice have required higher doses than humans in other studies due to higher surface area-to-body weight ratio. Thus, CBZ has significant potential to reverse hepatic fibrosis in A1ATD and is currently in phase II/III clinical trials for patients aged 15 years and older (NCT01379469). 


\section{Bile Acids as Small Molecule Therapy}

Bile acids are amphipathic steroid molecules normally synthesized in the liver from cholesterol, followed by excretion as the major component of bile. Synthetic bile acid agonists have recently emerged as promising agents in the treatment of chronic liver diseases, such as non-alcoholic fatty liver disease and primary biliary cirrhosis [59]. In general, most hydrophobic bile acids can have toxic effects, while hydrophilic bile acids are cytoprotective. In wild-type (WT) mice, common bile duct ligation (BDL), a model of obstructive cholestasis, alters bile acid composition, with significant increases in hydrophilic conjugated bile acids, causing pro-inflammatory but not cytotoxic effects in hepatocytes [60]. Interestingly, BDL has been shown to actually activate hepatoprotective mechanisms as well. In a series of elegant experiments, Gao et al. showed that activation of autophagy after BDL protects WT liver from cholestasis-induced injury, by eliminating accumulation of reactive oxygen species and preventing apoptosis [61]. Similarly, cultured human HepG2 cells pre-conditioned with sub-toxic concentrations of the hydrophobic bile acid glycochendeoxycholic acid (GCDCA) adapted towards further insult from GCDCA-induced apoptosis (a process called "hormesis") [62]. Khan et al. recently demonstrated that BDL accelerated clearance of ATZ globules in PiZ mice, with enhanced autophagy and proliferation of globule-devoid hepatocytes compared to globule-containing cells [63]. Comparable findings were also confirmed using a partial BDL model, in which the unligated right lobe served as an internal control for the ligated left lobe, supporting localized effects of retained bile acids in PiZ mouse liver [63]. These findings suggest a role for bile acid signaling as a potential therapeutic target in A1ATD.

The choleretic ursodeoxycholic acid (UDCA) has immunomodulatory, anti-oxidant, and anti-apoptotic properties, and it has long been a staple in the management of cholestasis, including in patients with mild-to-moderate liver disease in A1ATD [64]. In vitro treatment with the hydrophilic bile acid, tauroursodeoxycholic acid (TUDCA), has been shown to inhibit apoptosis in HEK293 cells over-expressing ATZ [65]. More recently, nor-ursodeoxycholic acid (nor-UDCA), a side-chain shortened derivative of UDCA, is another synthetic bile acid actively under investigation for the treatment of cholangiopathies, such as primary sclerosing cholangitis [66]. nor-UDCA is unique in that it is a weak acid that goes through cholehepatic shunting, with reabsorption directly from cholangiocytes to hepatocytes, to stimulate bile flow and remove toxic bile acids independently of known bile acid receptors [66] When PiZ mice were treated with this compound, there was a $32 \%$ increase in autophagic vacuoles with a significant reduction in the accumulation of ATZ globules. Furthermore, majority of globules were observed to be in continuity with an autophagic vacuole, suggesting a direct effect on enhancing autophagy. nor-UDCA also reduced both proliferation of inflammatory cells as well as the number and area of inflammatory foci. This was reflected in improved markers of hepatic injury and apoptosis, as well as less compensatory liver proliferation. Most interesting is that the effects of norUDCA seem to be unique to cells with the PiZ mutation and are not replicated in WT mice [67•]. Exogenous bile acids such as nor-UDCA are thus promising as small molecule therapy to increase autophagy and even protect against liver damage in A1ATD.

\section{Inhibiting Polymerization of ATZ}

Preventing the polymerization of insoluble ATZ in the liver is an attractive therapeutic approach. Homozygosity of the $Z$ allele (PiZZ) results from single base pair substitutions replacing lysine with glutamate at position 342 [68]. This change in negative-to-positive charge induces conformational changes that destabilize the protein's secondary structure, allowing unstable $\beta$-sheets to sequentially polymerize with exposed reactive loops, generating insoluble aggregates. The formation of hydrophobic pockets within the polymers has encouraged structure-based drug discovery. Through virtual ligand screening of over 1 million small molecules, Mallya et al. identified six compounds that could block the polymerization of ATZ in vitro [69]. These compounds targeted the "gap" between strands 3 and 5 of $\beta$-sheet $A$, which compromises the binding site for serpin inhibitory activity on neutrophil elastase. At least one of the small molecules (CG) was able to actively increase the clearance of ATZ polymers in vitro. When assayed against additional screening libraries, this lead compound was found to be highly selective and therefore less likely to have unwanted off-target effects. Subsequent structural studies of protein docking in vitro and in silico have identified other potential small molecule candidates, such as the nucleoside analog S-(4-nitrobenzyl)-6-thioguanosine, offering novel mechanisms to induce and model conformational changes using molecular dynamics [70].

Similar strategies have identified biologic small molecules, such as monoclonal antibodies, that can prevent ATZ polymer formation while preserving serine protease inhibitor activity $[71 \bullet \bullet$. Intrabodies are intracellularly expressed single chain antibody fragments (scFv), composed of one heavy (VH) and one light (VL) variable domains linked by a synthetic flexible peptide. The resulting $\mathrm{scFv}$ is the smallest fragment of an antibody capable of preserving antigen-binding specificity, while maintaining solubility and stability within the cell. Most importantly, scFv can be targeted to subcellular compartments by incorporating trafficking signals specific for the ER or other organelles, making them ideal candidates for correcting proteinopathies. For example, scFv4B12 is the isolated Fab domain from a mouse anti-human Z A1AT antibody, targeted to the ER by a C-terminal KDEL sequence. 
scFv4B12 inhibited the intracellular polymerization of ATZ by up to $60 \%$ in vitro, also confirming that most of the ATZ polymerization occurs within the ER [72]. This led to a significant improvement of ATZ secretion without generating additional ER stress. The ATZ protein also retained almost two-thirds of its inhibitory activity against neutrophil elastase in vitro. This partially restored serpin activity suggests that binding to scFv4B12 induces a substantially folded, nativelike form. Future directions would be to optimize the impact of this mechanism to completely eliminate the formation of any polymers and to develop a delivery mechanism for in vivo trials.

\section{Gene Therapy and Editing}

Several groups are actively pursuing genetic approaches to A1ATD therapy (see Table 1 for current clinical trials). An innovative strategy involves targeted "knock-down" of the mutant ATZ gene in hepatocytes. The creation of a second generation antisense oligonucleotide inhibitor that is complimentary to the mutant human AlAT gene (AAT-ASO, coding for the ATZ protein) resulted in a significant reduction in A1AT mRNA and protein levels in PiZ transgenic mice, which over-express human ATZ in the liver [73••]. This data was replicated in both young and adult PiZ mice to show that AAT-ASO both prevents and decreases ATZ protein aggregate formation, in addition to improving liver fibrosis. Administration in WT non-human primates also led to a significant reduction in levels of circulating normal A1AT, demonstrating long-term potential for this approach in higher species [74]. On a similar note, targeted gene correction with microRNA (miRNA) "dual therapy" has also been studied in PiZ mice, where effective knock-down of mutant ATZ is accompanied by expression of wild-type A1AT (ATM), inducing both decreased hepatic inflammation and ATZ globule accumulation, with concomitant increase in normal serum A1AT [75]. Clinical trials in adults are also ongoing for intramuscular (IM) injection of recombinant adenoassociated virus vectors expressing normal A1AT (rAAV1hAAT, Table 1). Initial reports demonstrated dose-dependent, sustained transgene expression of normal A1AT in the absence of significant adverse effects, up to 5 years after a single IM injection of rAAV1-hAAT [76].

While still in experimental stages, gene transfer may provide another targeted mechanism to induce autophagic clearance of the mutated ATZ protein. In pre-clinical studies, liverdirected gene transfer of transcription factor EB (TFEB), a master regulator of autophagy, reduced mutant ATZ globules in PIZ mice by enhancing autophagy [77]. There was an increase in fusion of autophagosomes with lysosomes, and more ATZ globules were observed within autolysosomes, corresponding to diminished ATZ globules in the cytoplasm. Histologically, there was also less fibrosis and apoptosis. The mice were observed for an additional 6 months on TFEB gene therapy with no visible signs of liver disease [77]. These preliminary results are promising and support trials for safety and efficacy in humans.

\section{Cell Therapy}

To date, there has only been identification of one human SNP in the SERPINA1 gene that confers susceptibility for liver disease A1ATD, and reporter gene assays failed to show a functional difference between alleles [33]. Without extensive knowledge of genetic modifiers that increase the risk of liver disease, stem cell therapy may be the key to developing more personalized approaches. Somatic stem cells from patients with A1ATD can be reprogrammed to induced pluripotent stem cells (iPSCs) which can generate hepatocyte-like cells (iHeps) [78, 79]. Although these cells do not function completely as native hepatocytes, they do maintain similar ultrastructure and secrete proteins unique to native hepatocytes. The iPSC model is distinct in the ability to differentiate cells from patients with severe liver disease from those that do

Table 1 Clinical studies listed in ClinicalTrials.gov for therapy of liver disease in A1ATD [87]

\begin{tabular}{lllll}
\hline Therapeutic agent & Therapeutic mechanism & Methodology & Clinical trial ID & Current status \\
\hline Carbamazepine (CBZ) & $\begin{array}{l}\text { Mood stabilizer and anti-epileptic drug that enhances } \\
\text { autophagy to increase ATZ protein clearance } \\
\text { AAV gene transfer of normal A1AT into muscle cells }\end{array}$ & Phase 2 & NCT01379469 & Recruiting \\
AAVrh.10 & RNAi-based knockout of ATZ protein expression & Phase 1 & NCT02168686 & Not open \\
ARC-AAT & AAV gene transfer of normal A1AT into muscle cells & Phase 1 & NCT00377416 & Active, not recruiting \\
$\begin{array}{l}\text { rAAV1-CB-hAAT } \\
\text { gene vector }\end{array}$ & RNAi-based knockout of ATZ protein expression & Phase 1/2 & NCT02503683 & Active, not recruiting \\
$\begin{array}{l}\text { ALN-AAT } \\
\text { rAAV1-CB-hAAT } \\
\text { gene vector }\end{array}$ & AAV gene transfer of normal A1AT into muscle cells & Phase 2 & NCT01054339 Completed \\
$\begin{array}{l}\text { 4-PBA } \\
\text { Molecular chaperone to increase ATZ protein secretion } \\
\text { from the liver }\end{array}$ & Phase 2 & NCT00067756 & Completed \\
\hline
\end{tabular}

This table does not include lung-targeted therapies (modified from Khan, 2016 [2]). 
not have clinically overt liver disease, despite sharing the same PiZZ mutation. Tafaleng et al. showed that patient-derived iHeps from those with severe liver disease processed the mutant ATZ protein slower and had increased globular inclusions in the ER, while iHeps from primarily lung-affected patients (liver "protected hosts") lacked such inclusions [80]. iHeps treated with lentiviral vector-mediated expression of short hairpin RNAs (shRNAs) directed against ATZ led to a $66 \%$ reduction in intracellular ATZ protein, which was functionally relevant and efficiently maintained during hepatocyte differentiation [81]. An emerging human genetic signature for susceptibility to liver disease was also recently described for A1ATD using iHeps [82]. On a similar note, activation of the c-JUN N-terminal kinase (JNK) pathway was recently found to aggravate proteotoxicity of mutant ATZ, and treatment of patient-derived iPSC with the JNK inhibitor SP600125 reduced ATZ polymer accumulation [83]. Since iHeps can model the variable clinical phenotypes of A1ATD, they may be used to develop future targets for therapy, ranging from disease modeling and drug screening to autologous cell transplant.

Stem cell therapy may prove to be superior to gene transfer because it provides a more patient-specific approach with ex vivo targeted gene correction. Recent advances in gene editing techniques, such as zinc finger nucleases (ZFN) and the CRISPR/ Cas9 system, in combination with delivery vectors engineered to target diseased tissue, could offer potential cures for A1ATD and many other monogenic liver-based metabolic diseases. Multiple patient-derived iPSC lines subjected to a combination of ZFN and piggyBac (a moth-derived DNA transposon) technology demonstrated bi-allelic correction of the PiZZ point mutation [84]. The modified cells continued to replicate and differentiate as expected, showing that this treatment did not impact their integrity. After differentiating into hepatocytes, the iPSC produced a properly formed A1AT with enzymatic inhibitory activity comparable to what is seen in healthy hepatocytes. Findings were replicated in vivo with the production of corrected human A1AT protein following transplantation into the liver of immunedeficient Alb-uPA ${ }^{+/+} / \operatorname{Rag}^{-/-} / I l 2 \mathrm{rg}^{-/}$mice, indicating successful engraftment. Results were consistent in cells derived from several patients; therefore, cell therapy could involve corrected patient-specific iPSC. Of concern, a small number of de novo mutations were detected during cell culture and gene editing, suggesting possible off-target effects. Routine screening of both primary and corrected iPSC lines would be critical for the safe use of differentiated stem cells in clinical applications.

Recently, mouse bone marrow-derived stem cells and human mesenchymal stem cells have also shown promising results, replacing ATZ globule-containing host hepatocytes when transplanted into PiZ mouse strains. Beneficial results included reduction in inflammatory response, fibrosis, and apoptotic death of hepatocytes, with improved hepatic glycogen storage and serum glucose levels [85]. In theory, cell infusions are by far safer, less invasive, and more cost effective than transplanting a whole organ; however, considerable variability exists among cell sources, preparations, and clinical protocols [86]. In practice, a single case report of human hepatocyte transplantation in a cirrhotic child with A1ATD resulted in orthotopic liver transplantation 2 days later, suggesting that cell therapy may be more appropriate as a "bridge" to organ transplant rather than a cure [87]. The successful use of safe and effective stem cell-derived therapies in large batches using uniform protocols is still under development. Clearly, there are many unanswered questions that must be addressed and optimized with both animal models and human trials before widespread clinical acceptance of liver cell therapy for A1ATD.

\section{Conclusions and Future Directions}

While the focus of this review has been on treatment of A1ATD-related liver disease, this only accounts for part of the morbidity, since removing the defective protein alone will not treat the lung disease. In fact, there is evidence that the minimal circulating ATZ protein may still protect lung function to some extent, as patients with "null" disease (producing no A1AT due to a premature stop codon) develop lung disease at an earlier age compared to other mutations [88]. Thus, some form of A1AT replacement therapy (protein or gene-directed) would still be necessary to restore serpin inhibitory activity in the lungs. To add to this complexity, a very recent study by Karadagi et al. reported that exogenous normal A1AT protein purified from human plasma can lower expression of the SERPINA1 gene in cultured primary human hepatocytes isolated from liver explants from patients with or without A1ATD [89]. Although liver biopsies were not available, similar trends were also detected in lung tissues obtained from a small cohort of PiZZ A1ATD emphysema patients receiving augmentation therapy relative to those without therapy [89]. These novel findings could redefine the role of augmentation therapy from merely replacing the deficient A1AT protein in the lungs to possibly down-regulating the toxic effects of mutant ATZ expression in the liver. Thus, the question of potential hepatoprotective effects for augmentation therapy in A1ATD is largely unexplored.

A1ATD is a common cause of genetic liver disease. It has a wide spectrum of presentations ranging from an incidental finding to end-stage cirrhosis requiring transplantation. There have been no reliable predictors identified for which patients will develop a more severe course of liver disease, making targeted individualized treatment an important consideration. At this time, curative therapies available are limited to transplantation, but research in this field has been very promising, revealing novel mechanisms of action. Several innovative small molecule and gene-based strategies are now in clinical trials, with an overall approach of reducing proteotoxicity and maintaining protein homeostasis in the liver. 
Acknowledgements Dr. Khan acknowledges grant support from NIH/ NICHD PHS K12HD052892, the Alpha-1 Foundation, and the Hillman Foundation. We also acknowledge the University of Pittsburgh's Center for Biologic Imaging Core (Dr. Donna Stolz), the Department of Pediatric Pathology (Dr. Sarangarajan Ranganathan), and the Pittsburgh Liver Research Center.

\section{Compliance with Ethical Standards}

Conflict of Interest The authors declare that they have no conflict of interest.

Human and Animal Rights and Informed Consent All reported studies with human or animal subjects performed by the authors have been previously published and complied with all applicable ethical standards, including national and institutional research standards and guidelines.

\section{References}

Papers of particular interest, published recently, have been highlighted as:

- Of importance

•• Of major importance

1. de Serres FJ. Alpha-1 antitrypsin deficiency is not a rare disease but a disease that is rarely diagnosed. Environ Health Perspect. 2003;111:1851-4.

2. Khan Z. Pathogenesis of alpha-1 antitrypsin deficiency in the liver: new approaches to old questions. J Liver Res Disord Ther. 2016;2: 00023.

3. Darlington GJ, Astrin KH, Muirhead SP, Desnick RJ, Smith M. Assignment of human alpha 1-antitrypsin to chromosome 14 by somatic cell hybrid analysis. Proc Natl Acad Sci U S A. 1982;79: 870-3.

4. DeMeo DL, Silverman EK. Alpha1-antitrypsin deficiency. 2: genetic aspects of alpha(1)-antitrypsin deficiency: phenotypes and genetic modifiers of emphysema risk. Thorax. 2004;59:259-64.

5. Ghouse R, Chu A, Wang Y, Perlmutter DH. Mysteries of alpha1antitrypsin deficiency: emerging therapeutic strategies for a challenging disease. Dis Model Mech. 2014;7:411-9.

6. Silverman EK, Sandhaus RA. Clinical practice. Alpha1-antitrypsin deficiency. N Engl J Med. 2009;360:2749-57.

7. Fagerhol MK, Laurell CB. The Pi system-inherited variants of serum alpha 1-antitrypsin. Prog Med Genet. 1970;7:96-111.

8. Fairbanks KD, Tavill AS. Liver disease in alpha 1-antitrypsin deficiency: a review. Am J Gastroenterol. 2008;103:2136-41. quiz 42

9. Greene CM, Miller SD, Carroll T, et al. Alpha-1 antitrypsin deficiency: a conformational disease associated with lung and liver manifestations. J Inherit Metab Dis. 2008;31:21-34.

10. Ekeowa UI, Gooptu B, Belorgey D, et al. Alpha1-antitrypsin deficiency, chronic obstructive pulmonary disease and the serpinopathies. Clin Sci (Lond). 2009;116:837-50.

11. Teckman JH, An JK, Blomenkamp K, Schmidt B, Perlmutter D. Mitochondrial autophagy and injury in the liver in alpha 1antitrypsin deficiency. Am J Physiol Gastrointest Liver Physiol. 2004;286:G851-62.

12. Chu AS, Perlmutter DH, Wang Y. Capitalizing on the autophagic response for treatment of liver disease caused by alpha-1-antitrypsin deficiency and other genetic diseases. Biomed Res Int. 2014;2014: 459823.
13. American Thoracic S, European Respiratory S. American Thoracic Society/European Respiratory Society statement: standards for the diagnosis and management of individuals with alpha-1 antitrypsin deficiency. Am J Respir Crit Care Med. 2003;168:818-900.

14. Khan Z, Venkat VL, Soltys KA, Stolz DB, Ranganathan S. A challenging case of severe infantile cholestasis in alpha-1 antitrypsin deficiency. Pediatr Dev Pathol. 2017;20:176-81.

15. Tretter JT. Adding alpha-1 antitrypsin deficiency to the newborn screen. J Pediatr Gastroenterol Nutr. 2015;60:e37.

16. Stockley RA, Dirksen A, Stolk J. Alpha-1 antitrypsin deficiency: the European experience. COPD. 2013;10(Suppl 1):50-3.

17. Campos MA, Wanner A, Zhang G, Sandhaus RA. Trends in the diagnosis of symptomatic patients with alpha1-antitrypsin deficiency between 1968 and 2003. Chest. 2005;128:1179-86.

18. Stoller JK, Sandhaus RA, Turino G, Dickson R, Rodgers K, Strange C. Delay in diagnosis of alpha1-antitrypsin deficiency: a continuing problem. Chest. 2005;128:1989-94.

19. Greulich T, Nell C, Herr C, et al. Results from a large targeted screening program for alpha-1-antitrypsin deficiency: 2003-2015. Orphanet J Rare Dis. 2016;11:75.

20. Sveger T. Liver disease in alpha1-antitrypsin deficiency detected by screening of 200,000 infants. N Engl J Med. 1976;294:1316-21.

21. Sveger T. Alpha 1-antitrypsin deficiency in early childhood. Pediatrics. 1978;62:22-5.

22. Piitulainen E, Carlson J, Ohlsson K, Sveger T. Alpha1-antitrypsin deficiency in 26-year-old subjects: lung, liver, and protease/ protease inhibitor studies. Chest. 2005;128:2076-81.

23. Teckman J, Pardee E, Howell RR, et al. Appropriateness of newborn screening for alpha1-antitrypsin deficiency. J Pediatr Gastroenterol Nutr. 2014;58:199-203.

24. Thelin T, McNeil TF, Aspegren-Jansson E, Sveger T. Identifying children at high somatic risk: parents' long-term emotional adjustment to their children's alpha 1 antitrypsin deficiency. Acta Psychiatr Scand. 1985;72:323-30.

25. Chu AS, Chopra KB, Perlmutter DH. Is severe progressive liver disease caused by alpha-1-antitrypsin deficiency more common in children or adults? Liver transplantation: official publication of the American Association for the Study of Liver Diseases and the International Liver Transplantation Society 2016.

26. Perlmutter DH, Chopra K, Chu A. Two new considerations for improving the diagnosis of alpha1-antitrypsin deficiency-associated liver disease. Dig Dis Sci. 2015;60:1511-3.

27. Potocnjak I, Tesovic G, Kuna AT, Stefanovic M, Zaja O. Unusually difficult clinical presentation of an infant suffering from congenital cytomegalovirus (CMV) infection combined with alpha 1antitrypsin (A1AT) deficiency. Biochemia medica. 2014;24:396402.

28. Topic A, Ljujic M, Radojkovic D. Alpha-1-antitrypsin in pathogenesis of hepatocellular carcinoma. Hepat Mon. 2012;12:e7042.

29. Gadek JE, Fells GA, Crystal RG. Cigarette smoking induces functional antiprotease deficiency in the lower respiratory tract of humans. Science. 1979;206:1315-6.

30. Piitulainen E, Tornling G, Eriksson S. Effect of age and occupational exposure to airway irritants on lung function in non-smoking individuals with alpha 1-antitrypsin deficiency (PiZZ). Thorax. 1997;52:244-8.

31. Janus ED, Phillips NT, Carrell RW. Smoking, lung function, and alpha 1-antitrypsin deficiency. Lancet. 1985;1:152-4.

32. Propst T, Propst A, Dietze O, Judmaier G, Braunsteiner H, Vogel W. High prevalence of viral infection in adults with homozygous and heterozygous alpha 1-antitrypsin deficiency and chronic liver disease. Ann Intern Med. 1992;117:641-5.

33. Chappell S, Hadzic N, Stockley R, Guetta-Baranes T, Morgan K, Kalsheker N. A polymorphism of the alpha1-antitrypsin gene represents a risk factor for liver disease. Hepatology. 2008;47:127-32. 
34. Pan S, Huang L, McPherson J, et al. Single nucleotide polymorphism-mediated translational suppression of endoplasmic reticulum mannosidase I modifies the onset of end-stage liver disease in alpha1-antitrypsin deficiency. Hepatology. 2009;50:27581.

35. Qu D, Teckman JH, Omura S, Perlmutter DH. Degradation of a mutant secretory protein, alpha1-antitrypsin Z, in the endoplasmic reticulum requires proteasome activity. J Biol Chem. 1996;271: 22791-5.

36. Teckman JH, Perlmutter DH. The endoplasmic reticulum degradation pathway for mutant secretory proteins alpha1-antitrypsin $\mathrm{Z}$ and $\mathrm{S}$ is distinct from that for an unassembled membrane protein. J Biol Chem. 1996;271:13215-20.

37. Wu Y, Whitman I, Molmenti E, Moore K, Hippenmeyer P, Perlmutter DH. A lag in intracellular degradation of mutant alpha 1-antitrypsin correlates with the liver disease phenotype in homozygous PiZZ alpha 1-antitrypsin deficiency. Proc Natl Acad Sci U S A. 1994;91:9014-8.

38. Rudnick DA, Liao Y, An JK, Muglia LJ, Perlmutter DH, Teckman $\mathrm{JH}$. Analyses of hepatocellular proliferation in a mouse model of alpha-1-antitrypsin deficiency. Hepatology. 2004;39:1048-55.

39. Clark VC. Liver transplantation in alpha-1 antitrypsin deficiency. Clinics in liver disease. 2017;21:355-65.

40. Kemmer N, Kaiser T, Zacharias V, Neff GW. Alpha-1-antitrypsin deficiency: outcomes after liver transplantation. Transplant Proc. 2008;40:1492-4.

41. Brusilow SW. Phenylacetylglutamine may replace urea as a vehicle for waste nitrogen excretion. Pediatr Res. 1991;29:147-50.

42. Maestri NE, Hauser ER, Bartholomew D, Brusilow SW. Prospective treatment of urea cycle disorders. J Pediatr. 1991;119: 923-8.

43. Brown CR, Hong-Brown LQ, Welch WJ. Correcting temperaturesensitive protein folding defects. J Clin Invest. 1997;99:1432-44.

44. Tamarappoo BK, Verkman AS. Defective aquaporin-2 trafficking in nephrogenic diabetes insipidus and correction by chemical chaperones. J Clin Invest. 1998;101:2257-67.

45. Rubenstein RC, Egan ME, Zeitlin PL. In vitro pharmacologic restoration of CFTR-mediated chloride transport with sodium 4phenylbutyrate in cystic fibrosis epithelial cells containing delta F508-CFTR. J Clin Invest. 1997;100:2457-65.

46. Tatzelt J, Prusiner SB, Welch WJ. Chemical chaperones interfere with the formation of scrapie prion protein. EMBO J. 1996;15: 6363-73.

47. Fan JQ, Ishii S, Asano N, Suzuki Y. Accelerated transport and maturation of lysosomal alpha-galactosidase A in Fabry lymphoblasts by an enzyme inhibitor. Nat Med. 1999;5:112-5.

48. Burrows JA, Willis LK, Perlmutter DH. Chemical chaperones mediate increased secretion of mutant alpha 1-antitrypsin (alpha 1-AT) $Z$ : a potential pharmacological strategy for prevention of liver injury and emphysema in alpha 1-AT deficiency. Proc Natl Acad Sci U S A. 2000;97:1796-801.

49. Teckman JH. Lack of effect of oral 4-phenylbutyrate on serum alpha-1-antitrypsin in patients with alpha-1-antitrypsin deficiency: a preliminary study. J Pediatr Gastroenterol Nutr. 2004;39:34-7.

50. Kaletta T, Hengartner MO. Finding function in novel targets: C. elegans as a model organism. Nat Rev Drug Discov. 2006;5: 387-98.

51. Silverman GA, Luke CJ, Bhatia SR, et al. Modeling molecular and cellular aspects of human disease using the nematode Caenorhabditis elegans. Pediatr Res. 2009;65:10-8.

52. Artal-Sanz M, de Jong L, Tavernarakis N. Caenorhabditis elegans: a versatile platform for drug discovery. Biotechnol J. 2006;1:140518.

53. Long OS, Benson JA, Kwak JH, et al. A C. elegans model of human alpha1-antitrypsin deficiency links components of the
RNAi pathway to misfolded protein turnover. Hum Mol Genet. 2014;23:5109-22.

54. Gosai SJ, Kwak JH, Luke CJ, et al. Automated high-content live animal drug screening using $C$. elegans expressing the aggregation prone serpin alpha1-antitrypsin Z. PLoS One. 2010;5:e15460.

55. Dubouloz F, Deloche O, Wanke V, Cameroni E, De Virgilio C. The TOR and EGO protein complexes orchestrate microautophagy in yeast. Mol Cell. 2005;19:15-26.

56. Paglin S, Lee NY, Nakar C, et al. Rapamycin-sensitive pathway regulates mitochondrial membrane potential, autophagy, and survival in irradiated MCF-7 cells. Cancer Res. 2005;65:11061-70.

57. Kaushal S, Annamali M, Blomenkamp K, et al. Rapamycin reduces intrahepatic alpha-1-antitrypsin mutant $Z$ protein polymers and liver injury in a mouse model. Exp Biol Med (Maywood). 2010;235: $700-9$.

58. Hidvegi T, Ewing M, Hale P, et al. An autophagy-enhancing drug promotes degradation of mutant alpha1-antitrypsin $\mathrm{Z}$ and reduces hepatic fibrosis. Science. 2010;329:229-32.

59. Arab JP, Karpen SJ, Dawson PA, Arrese M, Trauner M. Bile acids and nonalcoholic fatty liver disease: molecular insights and therapeutic perspectives. Hepatology. 2017;65:350-62.

60. Zhang Y, Hong JY, Rockwell CE, Copple BL, Jaeschke H, Klaassen CD. Effect of bile duct ligation on bile acid composition in mouse serum and liver. Liver Int: Off J Int Assoc Stud Liver. 2012;32:58-69.

61. Gao L, Lv G, Guo X, et al. Activation of autophagy protects against cholestasis-induced hepatic injury. Cell Biosci. 2014;4:47.

62. Verhaag EM, Buist-Homan M, Koehorst M, Groen AK, Moshage $\mathrm{H}$, Faber KN. Hormesis in cholestatic liver disease; preconditioning with low bile acid concentrations protects against bile acid-induced toxicity. PloS one. 2016;11:e0149782.

63. Khan Z, Yokota S, Ono Y, et al. Bile duct ligation induces ATZ globule clearance in a mouse model of alpha-1 antitrypsin deficiency. Gene Expr. 2017;17:115-27.

64. Lykavieris P, Ducot B, Lachaux A, et al. Liver disease associated with ZZ alpha1-antitrypsin deficiency and ursodeoxycholic acid therapy in children. J Pediatr Gastroenterol Nutr. 2008;47:623-9.

65. Miller SD, Greene CM, McLean C, et al. Tauroursodeoxycholic acid inhibits apoptosis induced by $\mathrm{Z}$ alpha-1 antitrypsin via inhibition of bad. Hepatology. 2007;46:496-503.

66. Glaser SS, Alpini G. Activation of the cholehepatic shunt as a potential therapy for primary sclerosing cholangitis. Hepatology. 2009;49:1795-7.

67. Tang Y, Fickert P, Trauner M, Marcus N, Blomenkamp K, Teckman J. Autophagy induced by exogenous bile acids is therapeutic in a model of alpha-1-AT deficiency liver disease. Am J Physiol Gastrointest Liver Physiol. 2016;311:G156-65. This study shows promising results that treatment with the bile acid nor-UDCA can induce autophagy and decrease ATZ globules in PiZ mice.

68. Ledley FD, Woo SL. Molecular basis of alpha 1-antitrypsin deficiency and its potential therapy by gene transfer. J Inherit Metab Dis. 1986;9(Suppl 1):85-91.

69. Mallya M, Phillips RL, Saldanha SA, et al. Small molecules block the polymerization of $\mathrm{Z}$ alpha1-antitrypsin and increase the clearance of intracellular aggregates. J Med Chem. 2007;50:5357-63.

70. Berthelier V, Harris JB, Estenson KN, Baudry J. Discovery of an inhibitor of Z-alpha1 antitrypsin polymerization. PLoS One. 2015;10

71.• Motamedi-Shad N, Jagger AM, Liedtke M, et al. An antibody that prevents serpin polymerisation acts by inducing a novel allosteric behaviour. The Biochemical Journal. 2016;473:3269-90. This study provides innovative data on use of antibodies as small molecule treatment for binding and correcting ATZ protein structure.

72. Ordonez A, Perez J, Tan L, et al. A single-chain variable fragment intrabody prevents intracellular polymerization of $\mathrm{Z}$ alpha1- 
antitrypsin while allowing its antiproteinase activity. FASEB J. 2015;29:2667-78.

73.• Guo S, Booten SL, Aghajan M, et al. Antisense oligonucleotide treatment ameliorates alpha-1 antitrypsin-related liver disease in mice. J Clin Invest. 2014;124:251-61. This study demonstrates effective gene editing in PiZ mice with ASO technology

74. Wooddell CI, Blomenkamp KS, Kanner S, Chu Q, Hamilton HL, Wakefield DH, et al. A hepatocyte-targeted RNAi-based treatment for liver disease associated with alpha-1-antitrypsin deficiency [Abstract]. Hepatology. 2014;60:249A.

75. Mueller C, Tang Q, Gruntman A, et al. Sustained miRNA-mediated knockdown of mutant AAT with simultaneous augmentation of wild-type AAT has minimal effect on global liver miRNA profiles. Molecular therapy: the journal of the American Society of Gene Therapy. 2012;20:590-600.

76. Mueller C, Gernoux G, Gruntman AM, et al. 5 year expression and neutrophil defect repair after gene therapy in alpha-1 antitrypsin deficiency. Molecular therapy: the journal of the American Society of Gene Therapy 2017.

77. Pastore N, Ballabio A, Brunetti-Pierri N. Autophagy master regulator TFEB induces clearance of toxic SERPINA1/alpha-1antitrypsin polymers. Autophagy. 2013;9:1094-6.

78. Rashid ST, Corbineau S, Hannan N, et al. Modeling inherited metabolic disorders of the liver using human induced pluripotent stem cells. J Clin Invest. 2010;120:3127-36.

79. Choi SM, Kim Y, Shim JS, et al. Efficient drug screening and gene correction for treating liver disease using patient-specific stem cells. Hepatology. 2013;57:2458-68.
80. Tafaleng EN, Chakraborty S, Han B, et al. Induced pluripotent stem cells model personalized variations in liver disease resulting from alpha1-antitrypsin deficiency. Hepatology. 2015;62:147-57.

81. Eggenschwiler R, Loya K, Wu G, et al. Sustained knockdown of a disease-causing gene in patient-specific induced pluripotent stem cells using lentiviral vector-based gene therapy. Stem Cells Transl Med. 2013;2:641-54.

82. Wilson AA, Ying L, Liesa M, et al. Emergence of a stage-dependent human liver disease signature with directed differentiation of alpha1 antitrypsin-deficient iPS cells. Stem cell reports. 2015;4:873-85.

83. Pastore N, Attanasio S, Granese B, et al. Activation of JNK pathway aggravates proteotoxicity of hepatic mutant $\mathrm{Z}$ alpha1antitrypsin. Hepatology 2017.

84. Yusa K, Rashid ST, Strick-Marchand H, et al. Targeted gene correction of alpha1-antitrypsin deficiency in induced pluripotent stem cells. Nature. 2011;478:391-4.

85. Baligar $\mathrm{P}$, Kochat V, Arindkar SK, et al. Bone marrow stem cell therapy partially ameliorates pathological consequences in livers of mice expressing mutant human alpha1-antitrypsin. Hepatology 2017.

86. Khan Z, Strom SC. Hepatocyte transplantation in special populations: clinical use in children. Methods Mol Biol. 2017;1506:3-16.

87. Strom SC, Chowdhury JR, Fox IJ. Hepatocyte transplantation for the treatment of human disease. Semin Liver Dis. 1999;19:39-48.

88. Fregonese L, Stolk J, Frants RR, Veldhuisen B. Alpha-1 antitrypsin null mutations and severity of emphysema. Respir Med. 2008;102:876-84.

89. Karadagi A, Johansson H, Zemack H, et al. Exogenous alpha 1antitrypsin down-regulates SERPINA1 expression. PLoS One. 2017;12:e0177279. 